\title{
AVALIAÇÃO DO CICLO DE VIDA ENERGÉTICO (ACVE) E DO DESEMPENHO TÉRMICO DE UMA HABITAÇÃO DE LIGHT STEEL FRAMING COM O USO DE DIFERENTES TIPOS DE ISOLANTES TÉRMICOS
}

\author{
Life Cycle Energy Assessment (LCEA) and Thermal Performance of a Light Steel \\ Framing Housing Using Different Types of Thermal Insulation
}

\author{
Lucas Rosse Caldas ${ }^{1}$, Rosa Maria Sposto ${ }^{2}$, Alexandre Mendonça Souto Lopes ${ }^{3}$, Werner Castro Tavares \\ Recebido em 03 de novembro de 2015; recebido para revisão em 09 de novembro de 2015; aceito em 11 de março \\ de 2016; disponível on-line em 20 de março de 2016.
}

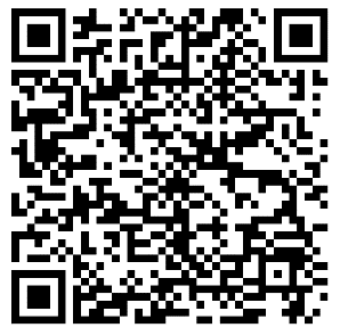

PALAVRAS CHAVE:

$\mathrm{ACV}$;

Desempenho térmico;

Light steel framing;

Isolantes térmicos.

KEYWORDS:

LCEA;

Thermal performance;

Light steel framing;

Thermal insulation.
RESUMO: Como forma de mensurar o consumo de energia ao longo do ciclo de vida dos diversos sistemas construtivos existentes, entre eles o light steel framing (LSF), tem sido aplicado a avaliação do ciclo de vida energético (ACVE). A ACVE foi aplicada em diversos estudos nacionais e internacionais, e no caso do LSF já foi verificado em alguns estudos nacionais. No entanto, ainda existe uma lacuna de estudos relacionados com o desempenho térmico e com os isolantes térmicos utilizados, principalmente por meio de simulações termoenergéticas. Neste sentido, o presente trabalho teve como objetivo avaliar a energia consumida ao longo do ciclo de vida de uma habitação de LSF, comparando o desempenho térmico deste sistema sem e com três isolantes térmicos, sendo eles: lã de vidro, lã de rocha e poliestireno expandido (EPS). A metodologia utilizada foi a pesquisa bibliográfica e simulação computacional em um software de simulação termoenergético. Ao final foi levantada a energia incorporada dos materiais utilizados nas fachadas, energia consumida nos transportes e energia gasta pelos equipamentos eletrônicos (energia operacional). A soma de todos estes consumos resultou na energia total, esta que foi maior para o sistema sem isolamento térmico e menor para o sistema com EPS. Neste sentido, a principal contribuição deste trabalho foi apresentar um critério de sustentabilidade energética para a especificação de isolantes térmicos para o sistema de LSF. Este critério poderá subsidiar, durante a etapa de projeto, a escolha do sistema mais vantajoso tanto do ponto de vista térmico como do consumo de energia ao longo do ciclo de vida da edificação.

ABSTRACT: As a way to measure the energy consumption over the life cycle of the various existing building systems, including light steel framing (LSF), has been applied to evaluate the life cycle energy assessment (LCEA). The LCEA was applied in several national and international studies, and in the case of LSF has already been verified in some national studies. However, there is still a lack of research related to thermal performance and thermal insulation used, mainly through thermal-energetic simulations. In this context, this study aimed to evaluate the energy consumed throughout the life cycle of a LSF house, comparing the thermal performance of this system without and with three thermal insulations, which are: glass wool, rock wool and polystyrene expanded (EPS). The methodology used was the bibliographical research and computer simulation on a thermal-energetic simulation software. It was assessed the embodied energy of the materials used on the facades, energy consumed in transport and energy consumed by electronic equipment (operating energy). The sum of all these resulted in total energy consumption, this one was higher for the system without thermal insulation and lower to the system with EPS. In this sense, the main contribution of this paper is to present an energy sustainability criteria for the specification of thermal insulation for the LSF system. This criteria can support, during the design stage, the choice of the most advantageous system in terms of the thermal performance and the energy consumption throughout the life cycle of the building.

\footnotetext{
* Contato com os autores:

${ }^{1}$ e-mail: Irc.ambiental@gmail.com ( L. R. Caldas )

2e-mail: rmsposto@unb.br (R.M. Sposto)

Engenheira Civil, Doutora, professora do Programa de Pós-Graduação em Estruturas e Construção Civil, UnB.

1 e-mail: alex.ecivil@hotmail.com ( A. M. S. Lopes )

Engenheiro Civil, Faculdade de Tecnologia, Departamento de Engenharia Civil e Ambiental, UnB.

2e-mail: wernner.tavares@gmail.com (W.C.Tavares )

Engenheiro Civil, Faculdade de Tecnologia, Departamento de Engenharia Civil e Ambiental, UnB.
}

Engenheiro Civil, Ambiental e Sanitarista, Mestrando, aluno do Programa de Pós-Graduação em Estruturas e Construção Civil, UnB. 


\section{INTRODUÇÃO}

O consumo de energia no Brasil tornou-se alvo de grande interesse nos últimos dois anos, principalmente devido a grave crise energética instalada no país. O setor da construção civil é apontado como um dos principais consumidores de recursos naturais e energia, causador de diversos impactos ambientais no mundo e no Brasil.

Materiais como cimento, aço, cerâmica e alumínio são considerados os principais consumidores de energia no setor (TAVARES, 2006). Este intenso consumo está relacionado ao processo produtivo destes materiais. Sabe-se que mo Brasil, a maior parte das edificações habitacionais ainda é realizada de forma convencional, com o emprego de estrutura reticulada de concreto armado e alvenaria de vedação de blocos cerâmicos e de concreto; estes processos possuem um baixo desenvolvimento tecnológico.

Como forma de racionalização da construção no setor de edificações habitacionais, surgiram nos últimos anos sistemas construtivos industrializados, com menor percentual de perdas e resíduos gerados, de maior rapidez de execução e com menor consumo de materiais ou massa. Um destes sistemas é o Light Steel Framing (LSF), que é um sistema construtivo inovador no Brasil (considera-se inovador aquele que não possui normas técnicas prescritivas nacionais aplicáveis). É constituído por estrutura de perfis de aço galvanizado formados a frio, que são utilizados para a composição de painéis estruturais e não estruturais, vigas, tesouras de telhado e demais componentes. Devido à industrialização deste sistema, é possível uma construção a seco (permite a minimização do uso de água na montagem do sistema) com grande rapidez de execução (FREITAS; CRASTO, 2006).

Embora existam muitas vantagens deste sistema, que reproduz os princípios da industrialização da construção, ainda existem alguns obstáculos a serem enfrentado, como por exemplo, o atendimento dos requisitos e critérios de desempenho exigidos nas normas técnicas brasileiras, principalmente relacionadas ao desempenho térmico.

De acordo com Gomes (2007), nas construções em LSF o isolamento térmico baseia-se no conceito de isolação multicamada, que consiste na combinação de placas leves de fechamento e o preenchimento do espaço entre os fechamentos com material isolante (lã de vidro, lã de rocha, EPS, etc.). Portanto, há a necessidade de se avaliar a necessidade do isolamento térmico para o sistema de LSF, e verificar qual dos tipos de isolantes é o mais adequado. Para a adoção do isolante a ser utilizado é necessário definir critérios de seleção, tais como o atendimento do desempenho térmico, os custos, a disponibilidade no mercado, a facilidade de execução e os impactos ambientais, incluindo a quantidade de energia incorporada, por exemplo.

Atualmente no Brasil nota-se a urgente necessidade dos projetistas especificarem materiais que atendam aos requisitos e critérios de desempenho necessários, mas que também levem em consideração aspectos energéticos. A quantificação de energia consumida durante o processo produtivo de um material e o seu impacto na etapa operacional da edificação também é necessária.

Por exemplo, é necessário saber se um material que consome maior quantidade de energia durante sua fabricação apresentará um desempenho térmico adequado na etapa de operação da edificação, e se no ciclo de vida total da edificação ocorrerá em uma diminuição da energia total. Tal avaliação já vem sendo estudada em vários países, incluindo o Brasil, e é denominada de avaliação do ciclo de vida energética (ACVE).

\section{OBJETIVOS}

O presente trabalho teve como objetivo empregar a ACVE para avaliar a energia consumida ao longo do ciclo de vida de uma habitação de Light Steel Framing (LSF), comparando o desempenho térmico deste sistema sem e com três tipos de isolantes térmicos, sendo eles: lã de vidro, lã de rocha e poliestireno expandido (EPS). 


\section{REVISÃO BIBLIOGRÁFICA}

\subsection{LIGHT STEEL FRAMING}

O LSF é composto por vários componentes e subsistemas. Esses subsistemas são a fundação, a estrutura, o isolamento termoacústico e os fechamentos interno e externo. Para que o sistema como um todo cumpra os requisitos de desempenho e funções para qual foi projetado é necessário que os subsistemas sejam compatíveis entre si e a utilização de materiais com a qualidade adequada (FREITAS; CRASTO, 2006; GOMES, 2007; CAMPOS, 2014). Foram resumidos, de acordo com Tamaki (2015), os principais materiais empregados no sistema de LSF:

- A estrutura composta pelos perfis leves de aço. As espessuras mais usuais são $0,80 \mathrm{~mm}, 0,95$ $\mathrm{mm}$ e $1,25 \mathrm{~mm}$, considerando o revestimento mínimo de zinco de $180 \mathrm{~g} / \mathrm{m}^{2}$;

- Para o fechamento externo, podem ser empregadas placas cimentícias e/ou de fibrocimento e siding, que pode ser vinílico (PVC), de madeira ou cimentício. A característica comum a todos é a rápida instalação, além da resistência à ação dos ventos, água e umidade. A placa cimentícia é bastante utilizada, composta de cimento, fibras de celulose e agregados, reforçada com tela de fibra de vidro em ambas as faces;

- O fechamento interno é constituído por chapas de gesso acartonado. As placas têm dimensões de acordo com o fabricante, e espessuras que variam de $6,5 \mathrm{~mm}, 9,5 \mathrm{~mm}, 12,5 \mathrm{~mm}$ e $15 \mathrm{~mm}$;

- As chapas de OSB são formadas por partículas de madeira orientadas e prensadas, e no LSF são instaladas entre o perfil de aço e o revestimento. O OSB é utilizado como reforço da parede, que pode ser no perímetro externo e, internamente, nas paredes que vão receber cargas diferenciadas;

- O isolamento térmico e acústico no LSF é o de multicamada: os espaços entre as placas são preenchidos por mantas isolantes. A mais adotada é a lã de vidro, normalmente com $50 \mathrm{~mm}$. A lã de rocha normalmente tem custo superior, no entanto, apresenta maiores valores de resistência térmica e acústica. Existem no mercado materiais como EPS, poliuretano, entre outros.

\subsection{DESEMPENHO TÉRMICO DAS VEDAÇÕES VERTICAIS E CONSUMO DE ENERGIA NAS HABITAÇÕES}

O sistema de vedação (verticais e horizontais), também denominado de envoltória é um dos principais elementos da edificação, responsável pela proteção do ambiente interior dos fatores ambientais externos (IWARO; MWASHA, 2013). As trocas de energia entre os meios exterior e interior da edificação tem como cerne a envoltória da edificação (LAMBERTS; DUTRA; PEREIRA, 2004).

A norma de desempenho, a NBR 15575-4 (ABNT, 2013) define a vedação vertical como as partes da edificação habitacional que limitam verticalmente a edificação e seus ambientes, podendo ser as fachadas, as paredes ou as divisórias internas.

Foi criada uma parte específica da norma, a parte 4, para este sistema, o que mostra a sua importância no desempenho de uma edificação. As exigências dos usuários referentes às vedações verticais contempladas na norma são as seguintes: desempenho estrutural, segurança contra incêndio, estanqueidade, desempenho térmico, acústico, durabilidade e manutenibilidade (NBR 15575-4, ABNT, 2013).

De acordo com Akutsu e Lopes (1988) o desempenho térmico pode ser definido como a interação entre a edificação e o ambiente térmico a que a mesma está inserida ou suas condições de exposição. É influenciado pelas condições climáticas como a temperatura, umidade do ar, velocidade e direção dos ventos, radiação solar; e condições de implantação como latitude, longitude, topografia, orientação solar com o ambiente vizinho à edificação, além dos materiais, que dão forma e dimensões da edificação. 
Esta norma define os requisitos e critérios para a verificação dos níveis mínimos de desempenho térmico necessários para as vedações verticais externas. Um dos requisitos existentes na norma é o de "Adequação as paredes externas", sendo que dois critérios necessitam ser atendidos, o da transmitância térmica (U) e capacidade térmica (Ct) das vedações externas.

A transmitância térmica pode ser definida como a capacidade de conduzir certa quantidade de calor por unidade de área e variação de temperatura (CBIC, 2013). É calculada como o inverso da resistência térmica total e é normalmente expressa em $\mathrm{W} / \mathrm{m}^{2}$.K. É o somatório do conjunto de resistências térmicas correspondentes a cada camada de um componente, elemento ou sistema. Está diretamente relacionada a espessura e condutividade térmica dos materiais que compõem as camadas (NBR 15220-2, ABNT, 2008).

A capacidade térmica pode ser definida como a quantidade de calor necessária para variar em uma unidade a temperatura de um sistema, normalmente expressa em kJ/m².K (CBIC, 2013). É calculada pelo somatório das capacidades térmicas de cada componente, elemento ou sistema.

De acordo com Lamberts, Dutra e Pereira (2004), a maior parte do consumo de eletricidade nas habitações é destinado a geladeira, chuveiros e iluminação. No entanto, nos últimos anos o sistema de condicionamento ambiental vem aumentando sua participação no consumo total, chegando a $20 \%$ na média nacional.

Segundo os mesmos autores, o crescente desenvolvimento social, a diminuição dos custos dos aparelhos de ar condicionado, e com a execução de edificações não adequadas ao clima, a aquisição destes aparelhos será cada vez mais frequente, resultando no aumento do consumo de energia elétrica pelo setor residencial.

Em muitos estudos, internacionais e nacionais, já foi verificada a economia de energia proporcionada pela melhoria do desempenho térmico das fachadas. A melhoria do desempenho térmico normalmente é realizada pela adição de isolantes térmicos (Rakhshan et al., 2013), vidros especiais (Kim, 2011) , fachadas especiais, como fachadas ventiladas (Maciel, 2013) e fachadas vivas (Oliveira, 2015), ou emprego de materiais convencionais com desempenho térmico adequados à localidade da edificação (HUBERMAN e PEARLMUTTER , 2008).

\subsection{AVALIAÇÃO DO CICLO DE VIDA ENERGÉTICO (ACVE) APLICADA ÀS HABITAÇÕES}

De acordo com Lacerda e Dias (2011), para atingir a sustentabilidade no setor da construção civil deve ser considerada na etapa de planejamento do projeto, uma gestão adequada da obra, por meio do emprego de materiais geradores de menor impacto ambiental, além da avaliação do ciclo de vida destes materiais.

A Avaliação de Ciclo de Vida (ACV) pode ser definida como a compilação e a avaliação das entradas e saídas e dos potenciais impactos ambientais de um produto ao longo do seu ciclo de vida, como consumo de energia primária, potencial de aquecimento global, eutrofização, acidificação das águas e etc. A ACV tem sido utilizada para a avaliação do desempenho ambiental de diversos processos e produtos, inclusive aqueles ligados ao setor da construção civil (NBR ISO 14040: ABNT, 2009; BRIBIAN et al., 2011).

Segundo Tavares (2006), devido à complexidade do estudo de uma ACV e a diversidade de impactos a serem mensurados, muitos estudos tem tido como principal foco a mensuração do consumo de energia ao longo do ciclo de vida das edificações o que originou o termo avaliação do ciclo de vida energético (ACVE). Na ACVE são contabilizados os consumos energéticos de um produto ou processo, incluindo a energia elétrica e combustíveis utilizados (CABEZA et al. 2014).

A ACVE aplicada ao setor da construção civil, especificamente no setor de edificações, para ser melhor compreendida e estudada normalmente é dividida em etapas. Esta divisão varia de autor para autor, no entanto, três etapas são essenciais, sendo elas a de pré-uso, uso, e pós-uso. A etapa de 
pré-uso consiste nas atividades realizadas desde a extração e processamento dos materiais utilizados até a finalização da execução da edificação. A etapa de uso consiste na energia gasta na operação da edificação e energia incorporada dos materiais e equipamentos utilizados na manutenção. $A$ etapa de pós-uso é aquela relacionada ao transporte e disposição final dos resíduos gerados ao longo do ciclo de vida da edificação (TAVARES, 2006; KIM, 2011; CABEZA et al. 2014)

No Brasil, observa-se uma série de estudos relacionados à ACVE aplicada ao setor de edificações, entre elas podem ser citados: Guimarães (1985), Tavares (2006), Maciel (2013), Pereira (2014) e Oliveira (2015). No contexto internacional, é um tema que também tem ganhado bastante atenção, com destaque para os seguintes estudos: ADALBERTH (1997), MITHRARATNE e VALE (2004), SARTORI E HESTNES (2007), RAMESH et al. (2010), PAULSEN e SPOSTO (2013) e WEN e NOOR (2015).

Os estudos de Huberman e Pearlmutter (2008), Kim (2011), Taborianski e Prado (2012), Nordby e Shea (2013), utilizaram a ACVE relacionada com o desempenho e conforto térmico das vedações. Alguns estudos focaram nos isolantes térmicos como o de Radhi e Sharples (2013), Rakhshan et al. (2013)e Rodrigues e Freire (2014). No Brasil, há uma carência de estudos de ACVE com foco nos isolantes térmicos.

Com relação ao LSF, alguns estudos já aplicaram a ACVE como forma de mensurar o consumo de energia e/ou emissões de $\mathrm{CO}_{2}$ deste sistema construtivo. Podem ser citados os trabalhos de NABUT NETO (2011), PALÁCIO (2013), CARMINATTI JÚNIOR (2012) e PEDROSO (2015). Todos englobaram em seus estudos a etapa de préuso, no entanto, em relação ao desempenho térmico, somente alguns deles, sendo que os dois últimos se limitaram aos cálculos da NBR 15220-2 (ABNT, 2008), sem avaliar o desempenho térmico por meio de alguma simulação termoenergética, ainda mais comparando diferentes isolantes térmicos. Portanto, observa-se uma lacuna, o que justifica o foco deste trabalho.

\section{METODOLOGIA}

\subsection{CARACTERIZAÇÃO DA HABITAÇÃO ESTUDADA}

O objeto de estudo abordado nesta pesquisa consiste em uma habitação de 45,64 $\mathrm{m}^{2}$ localizada na cidade de Brasília - DF $\left(-15,45^{\circ}\right.$, $\left.47,95^{\circ}\right)$, que se encontra na zona bioclimática 4 (NBR 15220-2, ABNT, 2008). A orientação da habitação foi escolhida de forma que a fachada frontal ficou para o leste e os dormitórios para o norte, ou seja, a condição mais desfavorável para a avaliação da temperatura interna no interior da habitação. Foi considerada que a habitação é ocupada por 3 pessoas.

Foram consideradas as seguintes especificações para a habitação estudada: esquadrias de alumínio com vidro liso de $6 \mathrm{~mm}$, portas de alumínio, piso da parte externa de concreto, piso da parte interna de placas cerâmicas, cobertura composta por uma laje de concreto armado de $10 \mathrm{~cm}$, com uma camada de ar de $10 \mathrm{~cm}$ sobre a laje e telhas de fibrocimento, acabamento em pintura branca PVA (interior) e acrílica (exterior). Quanto aos compartimentos, é composta por dois dormitórios, sala, cozinha, um banheiro e uma área de serviço externa, conforme a Figura 1.

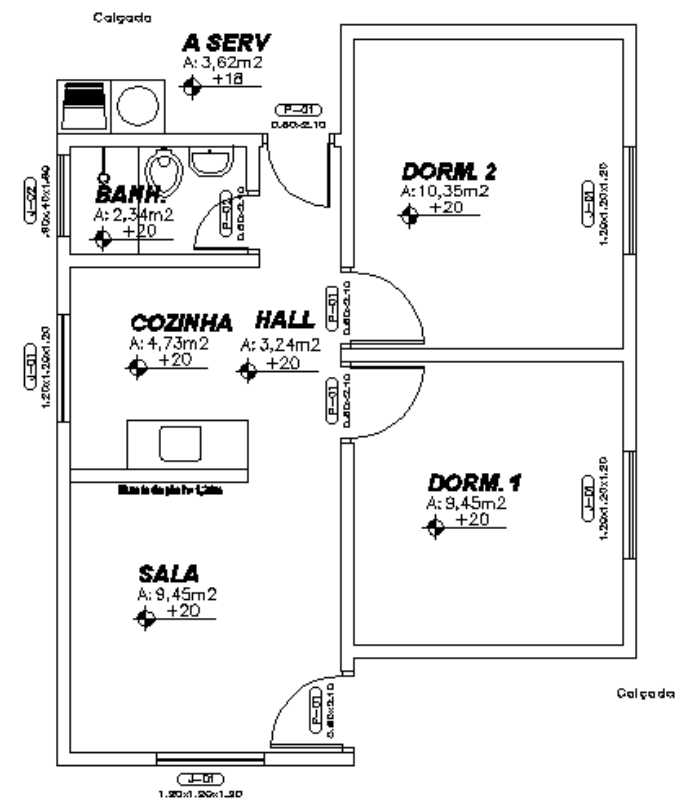

FIGURA 1: Planta baixa da habitação estudada. FONTE: PEDROSO (2015). 
O sistema de LSF avaliado é composto por perfis de aço tipo "U" (95 x 40 x 0,8 mm), com espaçamento entre os montantes de $40 \mathrm{~mm}$, contraventamento com chapa de OSB (11,3 mm), acabamento interno com placa de gesso acartonado (12,5 $\mathrm{mm}$ ) e acabamento externo com placa cimentícia $(10 \mathrm{~mm})$. Os isolantes considerados foram o poliestireno expandido (EPS), a lã de rocha e a lã de vidro, todos com a mesma espessura, de $50 \mathrm{~mm}$. Foram avaliadas quatro configurações do sistema de LSF, sendo elas (conforme a Figura 2):

- Sistema sem isolamento térmico (SS);

- Sistema com isolamento em EPS (SEPS);

- Sistema com isolamento de lã de rocha (SLR);

- Sistema com isolamento de lã de vidro (SLV).

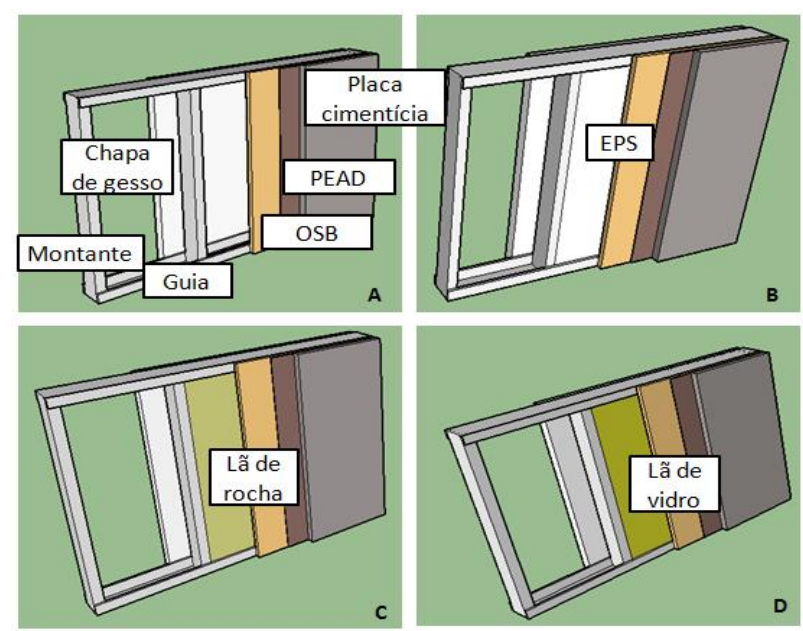

FIGURA 2: Configurações analisadas. (A) Configuração do sistema LSF sem isolante térmico. (B) Sistema LSF com EPS. (C) Sistema LSF com lã de rocha. (D) Sistema LSF com lã de vidro.

FONTE: Elaborada pelos autores.

\subsection{UNIDADE FUNCIONAL E ESCOPO DO ESTUDO}

De acordo com a NBR ISO 14040 (ABNT, 2009), a unidade funcional é o desempenho quantificado de um sistema de produto para ser utilizado como unidade de referência em um estudo de avaliação do ciclo de vida. Tem o objetivo de proporcionar referências para que as entradas e saídas estejam relacionadas, além de serem necessárias para assegurar a comparabilidade dos resultados entre dois ou mais elementos. A unidade funcional adotada neste trabalho foi a metragem quadrada $\left(\mathrm{m}^{2}\right)$ das fachadas da habitação, de $83,4 \mathrm{~m}^{2}$.
O escopo considerado no presente trabalho foi o consumo de energia oriundo das etapas de pré-uso e uso do ciclo de vida de uma habitação. A etapa de pós-uso foi desconsiderada devido a sua pequena participação, inferior a 3\%, como constatado nos estudos de TAVARES (2006), PAULSEN e SPOSTO (2013), e PEDROSO (2015). Foi considerada uma vida útil da habitação de 50 anos.

$\mathrm{Na}$ etapa de pré-uso foi considerada a energia incorporada da etapa de extração e processamento dos materiais (EI) e o consumo de energia referente à etapa de transporte dos materiais da fábrica até o canteiro de obras (ET). Foram levantadas as quantidades dos materiais constituintes das vedações verticais externas (fachadas) da habitação, em massa, para a posterior quantificação da energia incorporada. Os fatores de energia (FE) adotados foram retirados da literatura nacional e internacional. Para alguns materiais como a lã de rocha e lã de vidro, pela ausência de fontes nacionais confiáveis (visto que a maioria das fontes pesquisadas tratam estes dois materiais de forma similar, denominando-os por lã mineral); foram pesquisados ainda alguns environmental product declarations (EPDs), em um total de 3 EPDs de cada material e ao final foi realizada a média dos FE encontrados. As perdas dos materiais foram consideradas e foram retiradas da literatura nacional.

As distâncias utilizadas na etapa de transporte foram calculadas via Google Maps, considerando o transporte das fábricas até o canteiro de obras. Foi adotada a menor distância encontrada entre as fábricas dos materiais e o canteiro de obras. Como forma de limitar as fábricas a serem pesquisadas foram avaliadas aquelas sugeridas nos Documentos de Avaliação Técnica (DATecs $n^{\circ}$ 14, 15 e 16) que tratam do LSF. Foi utilizado o fator de consumo de energia no transporte, apresentado por Nabut Neto (2011), de $0,48 \mathrm{MJ} / \mathrm{km}$.t. Por fim, não foi considerada a etapa de execução da habitação visto que é uma habitação que demanda pequeno consumo de mão de obra e poucos equipamentos eletrônicos. Os dados utilizados estão apresentados conforme o Quadro 1. 
QUADRO 1: Quantidade de materiais, FE, perdas e distancias utilizadas.

\begin{tabular}{|c|c|c|c|c|c|}
\hline Materiais & $\begin{array}{c}\text { Quantidade } \\
\left(\mathrm{kg} / \mathrm{m}^{2}\right)\end{array}$ & FE (MJ/kg) & Fontes & $\begin{array}{c}\text { Perdas } \\
(\%)\end{array}$ & $\begin{array}{c}\text { Distância } \\
(\mathrm{Km})\end{array}$ \\
\hline Guias e Montantes (Aço) & 22,77 & 30 & Tavares (2006) & $10 \%$ & 16,9 \\
\hline Chapa de OSB (11, 1 $\mathrm{mm})$ & 8,26 & 7,5 & Tavares (2006) & $15 \%$ & 1298 \\
\hline Placa Cimentícia (10 mm) & 17 & 6 & Graf (2011) & $9 \%$ & 213 \\
\hline Placa de Gesso (12,5 mm) & 8 & 4,5 & Graf (2011) & $9 \%$ & 1195 \\
\hline PEAD & 0,1 & 95 & Tavares (2006) & $0 \%$ & 592 \\
\hline EPS (50 mm) & 0,1 & 112 & Tavares (2006) & $0 \%$ & 760 \\
\hline Lã de rocha $(50 \mathrm{~mm})$ & 3,5 & 16,54 & Média EPDs & $0 \%$ & 1065 \\
\hline Lã de vidro $(50 \mathrm{~mm})$ & 0,83 & 36,66 & Média EPDs & $0 \%$ & 1035 \\
\hline
\end{tabular}

${ }^{1}$ Perdas retiradas de Pedroso (2015).

Com relação à etapa de uso, foi considerada a etapa de operação da habitação (EO), que está relacionada ao uso dos equipamentos eletrônicos e cocção dos alimentos (conforme Quadro 2).

Para a adoção de equipamentos de ar condicionado (principal diferença no consumo de energia para diferentes desempenhos térmicos) considerou-se o desempenho e o conforto térmico apresentado pelos sistemas com os diferentes isolantes térmicos. O desempenho térmico foi calculado de duas formas:

- Procedimento de cálculo de acordo com as normas NBR 15575-4 (ABNT, 2013) e NBR 15220-2 (ABNT, 2008), por meio do cálculo de $U$ e Ct dos sistemas para a zona bioclimática 4, onde a cidade de Brasília-DF está localizada. Segundo a NBR 15575-4 (ABNT, 2013), o valor de $U$ deve ser menor ou igual a $3,7 \mathrm{~W} / \mathrm{m}^{2} . \mathrm{k}$ e para Ct é necessário atingir um valor maior ou igual a $130 \mathrm{kj} / \mathrm{m}^{2} . \mathrm{k}$;

- Utilização do software de simulação termoenergética Design Builder.

- A etapa de manutenção não foi considerada, visto que os isolantes térmicos se encontram no interior dos sistemas construtivos e só passarão por manutenção ou reposição após a substituição de um dos sistemas de revestimento (placas de gesso ou placas cimentícias).

QUADRO 2: Equipamentos eletrônicos adotados no consumo de energia da etapa operacional.

\begin{tabular}{|c|c|c|c|c|c|}
\hline $\begin{array}{l}\text { Equipamentos } \\
\text { Eletrônicos' }\end{array}$ & $n^{\circ}$ & $\begin{array}{l}\text { Pot Média } \\
\text { (Watts) }\end{array}$ & $\begin{array}{c}\text { Dias de uso no } \\
\text { mês }{ }^{1}\end{array}$ & $\begin{array}{l}\text { Tempo de utilização } \\
\text { (h/dia })\end{array}$ & $\begin{array}{l}\text { Consumo médio mensal } \\
\text { (kwh) }\end{array}$ \\
\hline Geladeira 1 porta & 1 & 35 & 30 & 24,0 & 25,20 \\
\hline Chuveiro Elétrico & 1 & 4500 & 30 & 0,50 & 67,50 \\
\hline Aparelho de DVD & 1 & 15 & 8 & 2,0 & 0,24 \\
\hline Lâmpadas & 2 & 100 & 30 & 5,0 & 30,00 \\
\hline Fogão elétrico & 1 & 2285 & 30 & 1,0 & 68,55 \\
\hline Lâmpadas & 3 & 60 & 30 & 5,0 & 27,00 \\
\hline Televisão 29" & 1 & 101 & 30 & 5,0 & 15,15 \\
\hline Ferro Elétrico & 1 & 1050 & 12 & 1,0 & 12,60 \\
\hline Lava Roupas & 1 & 147 & 12 & 1,0 & 1,76 \\
\hline Aparelho de som & 1 & 110 & 30 & 3,0 & 9,90 \\
\hline Telefone sem fio & 1 & 3 & 30 & 24,0 & 2,16 \\
\hline Computador & 1 & 63 & 30 & 8,0 & 15,12 \\
\hline Split no fresh air & 2 & - & - & - & $*$ \\
\hline \multicolumn{5}{|c|}{ Total (kwh) } & 275,80 \\
\hline
\end{tabular}

${ }^{1}$ Equipamentos eletrônicos retirado do Procel (2015).

*Consumo anual obtido na simulação termoenergética. 


\subsection{SIMULAÇÃO NO SOFTWARE DESIGN BUILDER}

Segundo Maciel (2013), o Design Builder possui integração com o software Energy Plus, ferramenta desenvolvida pelo Departamento de Energia dos EUA para quantificar o consumo energético de sistemas de resfriamento e aquecimento. Este é o software utilizado pelo Procel Edifica e por laboratórios credenciados no Brasil. O software foi utilizado para a avaliação do desempenho térmico e posteriormente para o cálculo do consumo de energia relacionado ao condicionamento ambiental da habitação.

Para a avaliação se os sistemas avaliados atendem o desempenho térmico exigido na NBR 15575-1 (ABNT, 2013) foram adotados os requisitos: "exigências de desempenho no verão" e "exigências de desempenho no inverno", com seus respectivos critérios, para o nível de desempenho mínimo (M). Para o verão o critério que deve ser atendido é que o valor máximo diário da temperatura do ar interior da habitação deve ser sempre menor ou igual o valor máximo diário de temperatura do ar exterior. Portanto, para o caso de Brasília, esta temperatura deve ser no máximo de $31,2^{\circ} \mathrm{C}$. Para o inverno a temperatura interna tem que ser maior que a externa mínima acrescida de $3^{\circ} \mathrm{C}$, o que para cidade de Brasília resultou em $14,23^{\circ} \mathrm{C}$ (NBR 15575-1, ABNT, 2013).

Nesta mesma norma é solicitado que sejam analisadas as temperaturas para os dias típicos de verão e inverno, no entanto, no presente trabalho foi realizada uma análise para as condições mais desfavoráveis para habitação. Para o verão foi utilizado o dia mais quente do ano no lugar do dia típico de verão e para o inverno dia mais frio do ano ao invés do dia típico do inverno. A justificativa para tal escolha foi o fato de que se as fachadas conseguirem atender os valores já definidos para os dias mais quente e mais frio do ano, certamente passará para os outros dias, inclusive os dias típicos.

Para o cálculo do consumo de energia foi necessário, primeiramente, definir a zona de conforto, que foi estipulada nos dados apresentados por Givoni (1992), variando de $18^{\circ} \mathrm{C}$ a $29^{\circ} \mathrm{C}$. Foi adotado este intervalo, visto que Givoni desenvolveu um estudo voltado para países de clima mais quente e em desenvolvimento, como é o caso do Brasil.

A partir da zona de conforto estabelecida, foram contabilizadas as horas de desconforto ao longo de 1 ano. Foram adotados dois critérios para a contabilização da zona de desconforto, sendo eles: (1) quando a temperatura no interior da habitação atinge valor superior a $29^{\circ} \mathrm{C}$. Não foram contabilizadas as horas que a temperatura é inferior a $18^{\circ} \mathrm{C}$, pois normalmente, para a realidade da cidade de Brasília, nestas situações as pessoas tentam melhorar as condições de conforto térmico pela troca de suas vestimentas; (2) só foram contabilizadas os horários das 17 às $07 \mathrm{~h}$ (dias da semana), visto que normalmente até este horário, os moradores não se encontram em suas residências e $07 \mathrm{~h}$ às $07 \mathrm{~h}$ (finais de semana).

É importante definir as horas de desconforto que foram contabilizadas, pois, são nestas horas que o sistema de resfriamento artificial é ativado, resultando no consumo de energia elétrica, na etapa operacional da habitação. Baseado no tamanho e características da habitação estudada, o sistema de resfriamento artificial adotado no presente trabalho foram 2 aparelhos de ar-condicionado tipo split no fresh air, sendo que cada aparelho ficou localizado em 1 dormitório. Foi considerada a eficiência (CoP) de 3,2, que corresponde ao selo A do Procel, portanto, o mais eficiente.

Os dados de entrada utilizados no software Design Builder foram baseados nas recomendações da NBR 15575-1 (ABNT, 2013) e as propriedades (densidade, calor específico e condutividade térmica) dos materiais utilizados foram retirados da 15220-2 (ABNT, 2008). Foram utilizados os dados metereológicos obtidos do Laboratório de Eficiência Energética em Edificações de Santa Catarina (LABEEE, 2015) para a cidade de Brasília - DF (BRA_BRASILIA_LABEEE). 


\section{RESULTADOS E DISCUSSÃO}

\subsection{ENERGIA INCORPORADA INICIAL (EI) E DA ETAPA DE TRANSPORTE (ET)}

A partir do levantamento da massa dos materiais das fachadas e dos $\mathrm{FE}$, juntamente com as distancias percorridas pelos materiais, foram obtidos os valores de El e ET conforme é apresentado nas Figuras 3 e 4, respectivamente.

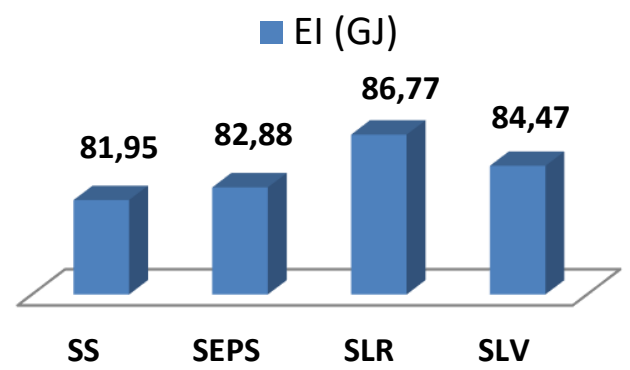

FIGURA 3: El dos sistemas avaliados.

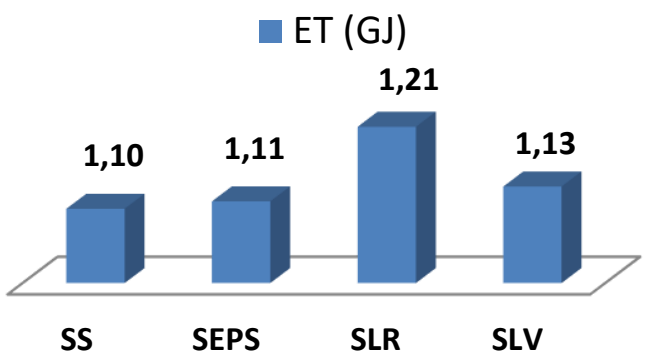

FIGURA 4: ET dos sistemas avaliados.

A partir da análise dos valores encontrados, é possível concluir:

- Os valores de EI são superiores aos valores de ET dos materiais;

- O sistema com isolante de lã de rocha (SLR) foi o que apresentou maior valor de El, uma diferença de $6 \%$ em relação ao sistema de referência (SS);

- Em relação a ET o sistema SLR também foi o que apresentou maior valor, no entanto a diferença foi maior em relação ao SS, aproximadamente $14 \%$.

Com relação à El, estes resultados são consequência dos FE adotados e da massa dos sistemas. O EPS apresenta um alto valor de FE, $112,0 \mathrm{MJ} / \mathrm{kg}$, no entanto, apresenta uma pequena participação em massa da fachada, somente $0,1 \mathrm{~kg} / \mathrm{m}^{2}$. A lã de rocha é o isolante que apresenta o menor $\mathrm{FE}$ entre os isolantes avaliados, de $16,5 \mathrm{MJ} / \mathrm{kg}$, no entanto, é o que apresenta maior massa, 3,5 kg/m². Por fim a lã de vidro apresenta o FE e massa intermediária, de $36,7 \mathrm{MJ} / \mathrm{kg}$ e $0,8 \mathrm{~kg} / \mathrm{m}^{2}$, o que no resultado final se mostrou maior que o SEPS. Portanto, em relação às configurações com isolantes térmicos, para o $\mathrm{El} O$ SEPS é o menos impactante do ponto de visto energético.

Com relação a ET, os valores encontrados são resultados da distância percorrida e a massa dos materiais. Portanto, quanto maior for estas duas variáveis, maior será o consumo de energia da etapa de transporte. O SLR possui a maior massa e foi o que apresentou a maior distância e assim foi o sistema que apresentou o maior valor de ET. Já o SEPS apresentou comportamento oposto, além de possuir a menor massa, apresentou a menor distância. Para a ET, entre as configurações com isolantes térmicos, o SEPS também foi o sistema mais indicado do ponto de vista energético. Cabe salientar que a distância influência de forma significativa o valor de ET, e, portanto, devem ser priorizadas fábricas mais próximas dos canteiros de obra.

\subsection{AVALIAÇÃO DO DESEMPENHO TÉRMICO}

Os resultados da simulação térmica, com e sem a presença dos isolantes térmicos, para os dias mais frio e quente do ano, estão apresentados nas Figuras 5 e 6, respectivamente. Os valores de $\mathrm{U}, \mathrm{Ct}$, temperatura máxima (tmax) e mínima (tmin) no interior da habitação para as configurações avaliadas estão apresentados conforme o Quadro 3.

A partir dos resultados obtidos pelo procedimento de cálculo simplificado e simulação computacional pode-se concluir uma divergência de informações. Os valores encontrados de $\mathrm{Ct}$, para todos as sistemas, estão abaixo do valor mínimo exigido pela norma, de $130 \mathrm{kj} / \mathrm{m}^{2}$.K. Já o valor de $U$ é menor que o valor máximo permitido, de $3,7 \mathrm{~W} / \mathrm{m}^{2} . \mathrm{k}$, portanto o critério da transmitância é atendido e o da capacidade não é atendido. É importante destacar a melhoria que a adição de isolantes térmicos proporcionou ao sistema de LSF, resultando em uma redução de $U$ de aproximadamente $80 \%$. Esta expressiva redução é 
resultado dos baixos valores de condutividade térmica dos isolantes térmicos.

Em relação aos resultados obtidos pela simulação computacional, todas as configurações (com isolantes e sem) atenderam os critérios de temperatura máxima e mínima definidos na norma NBR 155751-1 (ABNT, 2013), para a cidade de Brasília. No entanto, é possível perceber que a configuração sem isolante térmico apresenta um desempenho térmico inferior que os sistemas com isolantes térmicos. Tal resultado foi consequência da melhoria da transmitância térmica proporcionada pelos isolantes, já que a capacidade térmica dos materiais não apresentou uma grande variação.

Quando se comparam as três configurações com isolantes, é possível observar que todos apresentaram desempenho térmico semelhante. Destaca-se que a configuração com lã de rocha obteve os melhores resultados, tanto para condição de verão, como para condição de inverno. No entanto, esta diferença foi desprezível, considerando a avaliação do impacto do desempenho térmico no consumo de energia relacionado ao condicionamento ambiental.

Com relação ao conforto térmico, definido pela faixa de conforto entre 18 e $29^{\circ} \mathrm{C}$, as três configurações com isolantes não ultrapassaram o valor máximo, e também não apresentaram diferenças no consumo de energia pelos aparelhos de ar condicionado. Já o sistema sem isolantes (SS) não atendeu as questões de conforto nem para o verão e nem para o inverno, e como consequência foi contabilizado o consumo de energia do sistema de resfriamento, por meio da quantificação das horas de desconforto, respeitando os 2 critérios definidos no item de Metodologia. Foi quantificado o consumo de energia dos aparelhos de ar condicionado, resultando em um consumo anual de 175,64 kWh.

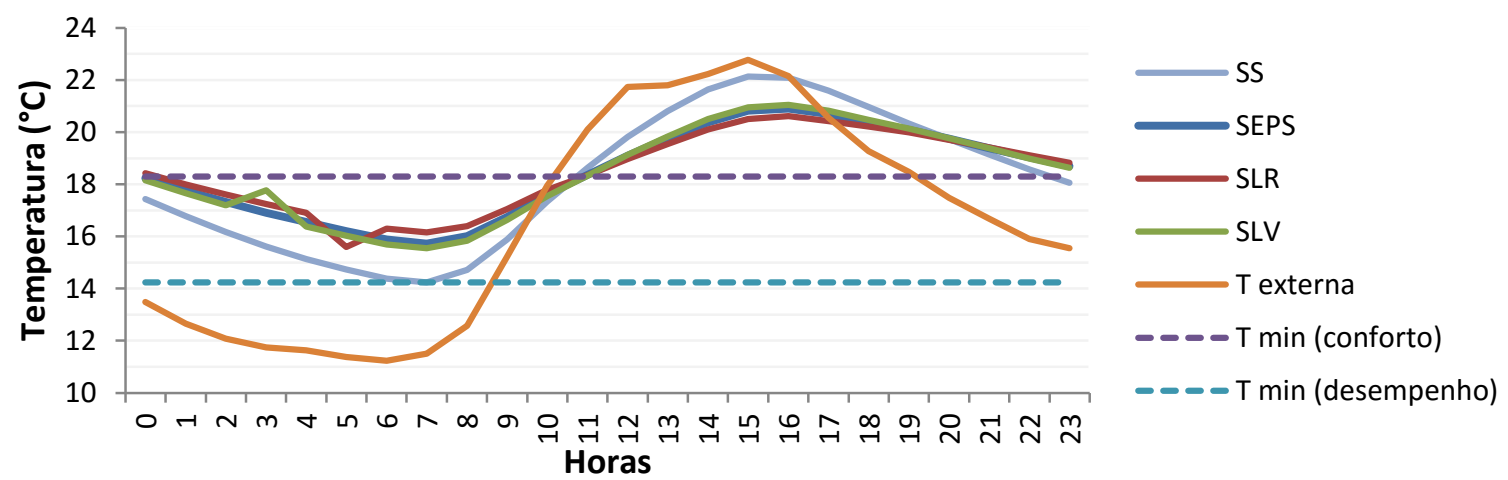

FIGURA 5: Variação de temperatura no dia mais frio do ano. SS - sistema sem isolamento térmico; SEPS sistema com isolamento de EPS; SLR - sistema com isolamento de lã de rocha; SLV - sistema com isolamento de lã de vidro; T externa - temperatura externa à edificação; T min (conforto) - temperatura mínima de conforto segundo Givoni (1992); Tmin (desempenho) - temperatura mínima para atingir o valor definido na NBR 15571-1 (ABNT, 2013).

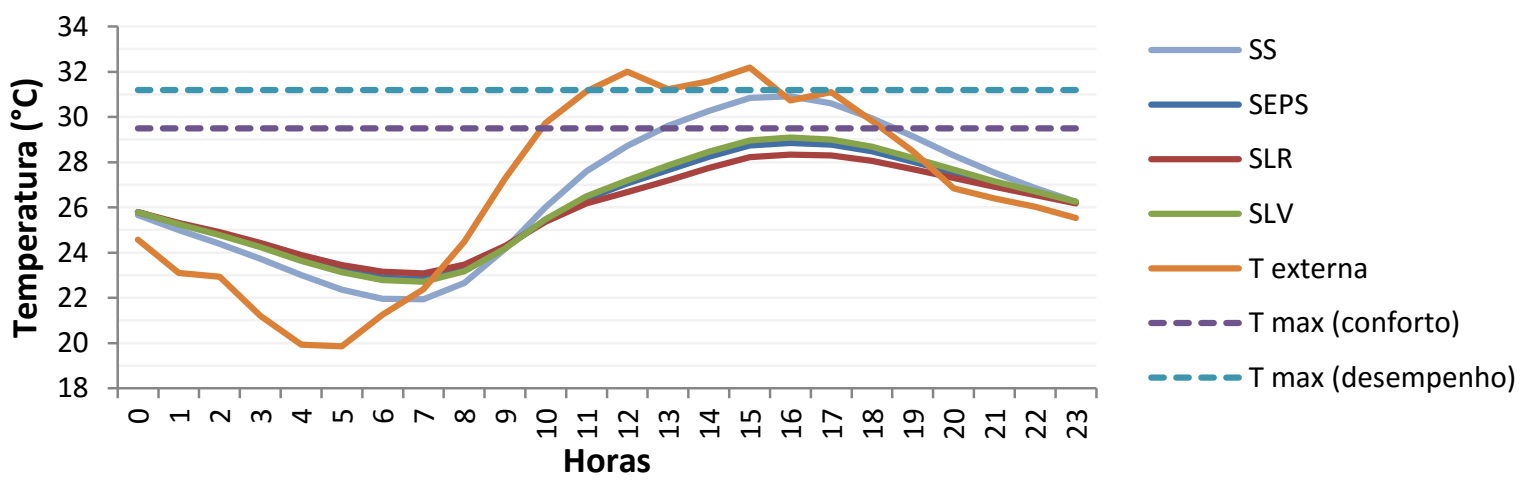

FIGURA 6: Variação de temperatura no dia mais quente do ano. T max (conforto) - temperatura máxima de conforto segundo Givoni (1992); Tmax (desempenho) - temperatura máxima para atingir o valor definido na NBR 15571-1 (ABNT, 2013). 


\section{QUADRO 3: Valores de U, Ct e temperaturas máxima e mínima para os sistemas avaliados}

\begin{tabular}{|c|c|c|c|c|}
\hline Sistemas & $\mathrm{U}\left(\mathrm{W} / \mathrm{m}^{2} \cdot \mathbf{k}\right)$ & $\mathrm{Ct}\left(\mathbf{k j} / \mathrm{m}^{2} \cdot \mathbf{k}\right)$ & $\mathrm{T}_{\max }\left({ }^{\circ} \mathrm{C}\right)$ & $\mathrm{T}_{\min }\left({ }^{\circ} \mathrm{C}\right)$ \\
\hline SS & 3,35 & 41,04 & 30,92 & 14,24 \\
\hline SEPS & 0,63 & 43,49 & 28,85 & 15,73 \\
\hline SLR & 0,64 & 44,05 & 28,33 & 16,15 \\
\hline SLV & 0,81 & 43,15 & 28,95 & 15,54 \\
\hline
\end{tabular}

\subsection{ENERGIA OPERACIONAL (EO)}

Como descrito na metodologia, para os horários onde a temperatura ultrapassou a temperatura de $29^{\circ} \mathrm{C}$, o aparelho de ar condicionado foi ligado, e, portanto, a energia consumida nestes horários foi contabilizada na energia operacional, juntamente com os outros equipamentos eletrônicos. Isto ocorreu somente para o caso sem isolante térmico (SS), e desta forma foram obtidos os valores de energia operacional (EO), conforme é apresentado na Figura 7.

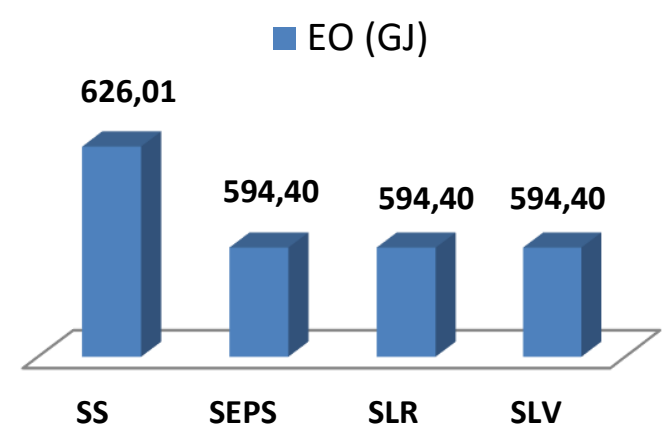

FIGURA 7: EO dos sistemas avaliados.

A partir dos valores encontrados, observa-se que as configurações com os isolantes apresentaram a mesma energia operacional, já que não apresentaram a necessidade de aparelho de ar condicionado, para as condições de conforto térmico estipuladas neste estudo. No entanto, a configuração sem isolante térmico, apresentou um acréscimo de energia operacional, aproximadamente $4 \%$. Comparando a EO com a EI e ET, os valores apresentados são superiores, cerca de 7 vezes superior a El e 500 vezes superior a ET. A prevalência da etapa operacional no ciclo de vida das habitações, acima de $60 \%$ da energia total, já foi resultado de muitos estudos nacionais e interacionais como o de Tavares(2006),
SARTORI e HESTNES (2007), PAULSEN e SPOSTO (2013), RAKHSHAN et al. (2013). Portanto, deve ser a etapa de maior foco dos projetistas.

\subsection{ENERGIA OPERACIONAL (EO)}

Foram encontrados os valores de ETOT, conforme é apresentado na Figura 8. É possível observar que a configuração SS foi a que apresentou maior energia total e a configuração SEPS a menor. As três configurações com isolantes apresentaram valores próximos, com uma diferença menor que 0,5\%. Comparando a diferença dos sistemas com isolantes e o de referência, a diferença foi de aproximadamente $4 \%$, como o resultado da EO. Tal resultado é mais uma evidencia da grande importância da EO em relação à ETOT.

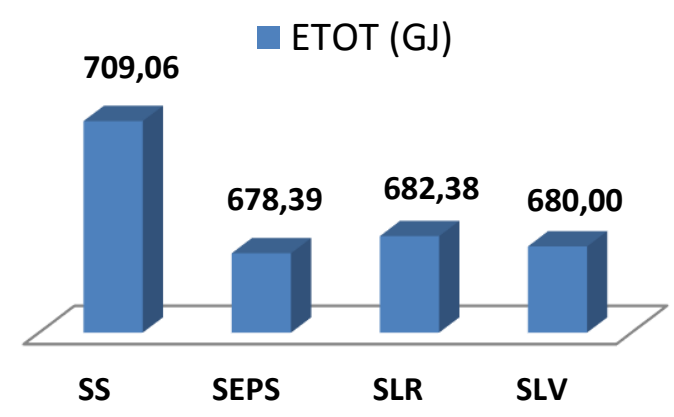

FIGURA 8: ETOT dos sistemas avaliados.

Sartori e Hestnes (2007) avaliaram 60 casos, em que a ACVE foi utilizada como ferramenta de contabilização e avaliação do consumo de energia ao longo do ciclo de vida de edificações. Concluíram que o aumento na El, na maioria dos casos, resulta na diminuição da EO e consequente diminuição da ETOT. Tal resultado foi constatado no presente trabalho, embora a adição de isolantes térmicos acarrete no aumento da El, a EO e ETOT tiveram uma redução. 


\section{CONCLUSÕES}

Foi realizada a avaliação do ciclo de vida energético (ACVE) e avaliação do desempenho térmico de uma habitação de LSF com 4 configurações de fachadas, com EPS, lã de rocha e lã de vidro, sendo a de referência sem isolante térmico. Com relação ao desempenho térmico, foram realizados dois procedimentos descritos na NBR 15575-1 (ABNT, 2013), o cálculo da transmitância e capacidade térmica e a simulação termoenergética no software Design Builder. Para o primeiro procedimento, nenhuma das configurações atendeu o critério mínimo de capacidade térmica. No entanto, para a simulação computacional, todos os sistemas apresentaram desempenho térmico adequado para o nível mínimo exigido na norma. A configuração sem isolante apresentou o pior desempenho térmico e a com lã de rocha o melhor desempenho.

Com relação ao consumo de energia ao longo do ciclo de vida da habitação, foram consideradas as etapas de extração, processamento e transporte dos materiais e a etapa de operação da habitação. A configuração com lã de rocha apresentou os maiores valores de El (86,77 GJ) e ET (1,21GJ). Para energia operacional a configuração de referência apresentou o maior consumo de energia e isto ocorreu devido à energia consumida no sistema de resfriamento utilizado para satisfazer a zona de conforto térmica estabelecida (máxima de $29^{\circ} \mathrm{C}$ ). Ao final, a ETOT foi maior para o sistema de referencia $(709,06 \mathrm{GJ})$, e menor para a configuração com EPS (678,39 GJ). Tal resultado mostrou que é vantajoso do ponto de vista energético, ao longo do ciclo de vida da habitação, adicionar isolantes térmicos, já que a etapa operacional se mostrou a mais impactante ao longo do ciclo de vida adotado de 50 anos.

$O$ presente trabalho avaliou diferentes configurações do LSF, que vem sendo cada vez mais empregado no Brasil, no contexto do desempenho térmico e consumo de energia, avaliando qual configuração do sistema é mais vantajosa a partir destes dois critérios.

Desta forma, a principal contribuição deste trabalho para a área do conhecimento foi apresentar um critério de sustentabilidade energética para a especificação de isolantes térmicos para o sistema de LSF, podendo subsidiar, durante a etapa de projeto, a escolha do sistema mais vantajoso tanto do ponto de vista térmico como energético.

Como sugestão para trabalhos futuros, podem ser avaliados outros impactos ambientais como emissões de $\mathrm{CO}_{2}$, consumo de água, geração de resíduos e etc; outros requisitos de desempenho como o acústico e qual o impacto da escolha dos isolantes com base nos custos.

\section{REFERÊNCIAS BIBLIOGRÁFICAS}

ADALBERTH, K. Energy use during the life cycle of buildings: A method. Building and Environment. v.32. p. 321 - 329. 1997.

AKUTSU, M.; LOPES, D. Simulação do desempenho térmico de edificações. In: Instituto de Pesquisas Tecnológicas do Estado de São Paulo - IPT. Tecnologia das Edificações. São Paulo: PINI, 1988.

ASSOCIAÇÃO BRASILEIRA DE NORMAS TÉCNICAS, NBR ISO 14040: Gestão ambiental - Avaliação do ciclo de vida - Princípios e estrutura. Rio de Janeiro, 2009.

NBR 15220-2. Desempenho Térmico de Edificações. Parte 2: Métodos de cálculo da transmitância térmica, da capacidade térmica, do atraso térmico e do fator solar de elementos e componentes de edificações. Rio de Janeiro, 2008.

NBR 15575-1. Edificações Habitacionais Desempenho. Requisitos Gerais. Rio de Janeiro, 2013.

NBR 15575-4. Edificações Habitacionais Desempenho. Sistemas de vedações verticais internas e externas - SVVIE. Rio de Janeiro, 2013.

BRIBIÁN, I. Z.; CAPILLA, A. V.; USÓN, A. A. Life cycle assessment of building materials: Comparative analysis of energy and environmental impacts and evaluation of the eco-efficiency improvement potential. Building and Environment. p. 1133-1140. 2011

CABEZA, L. F.; RINCÓN, L.; VILARIÑO, V.; PÉREZ, G.; CASTELL, A. Life cycle assessment(LCA) and life cycle energy analysis(LCEA) of buildings and the building sector : A review. Renewable and Sustainable Energy Reviews. p. 394-416. 2014.

CAMARA BRASILEIRA DA INDÚSTRIA DA CONSTRUÇÃO (CBIC). Desempenho de edificações habitacionais. Guia orientativo para atendimento a NBR 15575. Brasília, 2013. 
CAMPOS, P.F. Light Steel Framing - Uso em Construções Habitacionais Empregando a Modelagem Virtual como Processo e Planejamento. 198 p. Dissertação (Mestrado em Arquitetura e Urbanismo). Faculdade de Arquitetura e Urbanismo. Universidade de São Paulo, São Paulo, 2014.

CARMINATTI JÚNIOR, R. Análise do Ciclo de Vida Energético de Projeto de Habitações de Interesse Social Concebido em Light Steel Framing. Dissertação (Mestrado em Construção Civil) - Programa de PósGraduação em Construção Civil, Universidade de São Carlos, São Carlos, 2012.

FREITAS, A. M. S.; CRASTO, R. C. M. Steel Framing: Arquitetura. Instituto Brasileiro de Siderurgia. Centro Brasileiro da Construção em Aço. Rio de Janeiro. 2006.

GIVONI, B. Comfort climate analysis and building design guidelines. Energy and Buildings, v. 18, p. 11-23.1992.

GOMES, A. P. Avaliação do Desempenho Térmico de Edificações Unifamiliares em Light Steel Framing. 172p. Dissertação (Mestrado em Engenharia Civil). Escola de Minas em Engenharia Civil. Universidade Federal de Ouro Preto Ouro Preto, 2007.

GUIMARÃES, G.D. Análise Energética na Construção de Habitações. 1985. Dissertação (Mestrado em Engenharia Nuclear e Planejamento Energético). Universidade Federal do Rio de Janeiro, Rio de Janeiro, 1985.

HUBERMAN, N.; PEARLMUTTER, D. A life-cycle energy analysis of building materials in the Negev desert. Energy and Buildings. p. 837-848. 2008.

IWARO, J.; MWASHA, A. The impact of sustainable building envelope design on building sustainability using Integrated Performance Model. International Journal of Sustainable Built Environment. v.2. p. 153 171. 2013.

KIM, K. H. A comparative life cycle assessment of a transparent composite façade system and a glass curtain wall system. Energy and Buildings. p. 3436 -3445. 2011.

LABORATÓRIO DE EFICIÊNCIA ENERGÉTICA (LABEEE). Disponível em: http://www.labeee.ufsc.br/downloads/arquivosclimáticos/formato-epw. Acesso em 02/03/2015.

LACERDA, B.; DIAS, J. F. Análise Preliminar do Plano Diretor do Campus Glória Frente às Diretrizes LEED. Ciência e Engenharia. 20 (2): 21 - 30, jul. - dez. 2011.

LAMBERTS, R.; DUTRA, L.; PEREIRA, F. O. R. Eficiência Energética na Arquitetura. 2a ed. São Paulo: Pro Livros, 2004.
MACIEL, A.C.F. Energia incorporada de fachadas ventiladas. Estudo de caso para edificação habitacional em Brasília-DF. 2013. Dissertação (Mestrado em Estruturas e Construção Civil) - Faculdade de Tecnologia - Departamento de Engenharia Civil e Ambiental, Universidade de Brasília, Brasília, 2013.

MITHRARATNE, N.; VALE, B. Life cycle analysis model for New Zelands houses. Building and Environment.v. 39, p. 483-492, 2004.

NABUT NETO, A.C. Energia Incorporada e Emissões de CO2 de Fachadas. Estudo de Caso do Steel Frame para Utilização em Brasília. 154p. Dissertação (Mestrado em Estruturas e Construção Civil) - Faculdade de Tecnologia, Departamento de Engenharia Civil e Ambiental, Universidade de Brasília, Brasília, 2011.

NORDBY, A. S.; SHEA, A. D. Building materials in the operational phase- impacts of direct carbon exchanges and hygrothermal effects. Journal of Industrial Ecology.v. 17, n. 5, p. 763-776, 2013.

OLIVEIRA, L. B. A influência do sistema de fachada viva em manta no ciclo de vida energético de uma residência unifamiliar em clima tropical. 2015. Dissertação (Mestrado) - Programa de Pós-Graduação em Construção Civil, Universidade Federal do Paraná, Curitiba, 2015.

PALÁCIO, C.D.U. Energia incorporada de vedações para habitação de interesse social considerando-se o desempenho térmico. Estudo de caso com utilização do Light Steel Frame no Entorno do DF. 2013. Dissertação (Mestrado em Estruturas e Construção Civil) Departamento de Engenharia Civil e Ambiental, Universidade de Brasília, Brasília, 2013.

PAULSEN, J. S.; SPOSTO, R. M. A life cycle energy analysis of social housing in Brazil: Case Study for the program “MY HOUSE MY LIFE". Energy and Buildings. v. 57. p. 95102. 2013.

PEDROSO, G. M. P. Avaliação do Ciclo de Vida Energético (ACVE) de Sistemas de Vedação de Habitações. 2015. Tese (Doutorado) - Programa de PósGraduação em Estruturas e Construção Civil, Universidade de Brasília, Brasília, 2015.

PEREIRA, M.F. Conteúdo energético e emissões de CO2 em coberturas verdes, de telha cerâmica e de fibrocimento: estudo de caso. 2014. Dissertação (Mestrado) - Centro de Tecnologia, Programa de PósGraduação em Engenharia Civil, Universidade Federal de Santa Maria, Santa Maria, 2014. 
PROGRAMA NACIONAL DE CONSERVAÇÃO DE ENERGIA

(Procel). Disponível em:

<http://www.procelinfo.com.br/main.asp?View=\%7BE6

BC2A5F-E787-48AF-B485-439862B17000\%7D>.Acesso

em 24/05/15.

OLIVEIRA, L. B. A influência do sistema de fachada viva em manta no ciclo de vida energético de uma residência unifamiliar em clima tropical. 2015. Dissertação (Mestrado em Construção Civil) - Programa de PósGraduação em Construção Civil, Universidade Federal do Paraná, Curitiba, 2015.

RADHI, H.;SHARPLES, S. Global warming implications of façade parameters: a life cycle assessment of residential buildings in Bahrain. nvironmental Impact Assessment Review. v.38. p. 99- 108. 2013.

RAKHSHAN, K.; FRIESS, W. A.; TAJERZADEH, S. Evaluating the sustainability of improved building insulation: $A$ case study in the Dubai residential environment. Building and Environment, v. 67, p. 105-110. 2013.

RAMESH, T.; PRAKASH, R.; SHUKLA, K. K. Life cycle energy analysis of buildings: An overview. Energy and Buildings,.v.42. p. 1592 - 1600. 2010.

RODRIGUES, C.; FREIRE, C. Integrated life-cycle assessment and thermal dynamic simulation of alternative scenarios for the retrofit of a house. Building and Environment, v. 81, p. 105-110. 2014.

SARTORI, I.;HESTNES, A. G. Energy Use In The Life Cycle of Conventional and Low - Energy Buildings: A Review Article. Energy and Buildings. v.39. p. 249 - 257. 2007.

TABORIANSKI, V. M.; PRADO, R. T. A. Methodology of $\mathrm{CO} 2$ emission in the life cycle of office building façades. Environmental Impact Assessment Review. Science Direct. v. 33. p. 41 -47. 2012.

TAMAKI, L. Saiba como escolher componentes do sistema light steel framing. Téchne. Tecnologia. ed. 222, Setembro, 2015.

TAVARES, S. F. Metodologia de Análise do Ciclo de Vida Energética de Edificações Residenciais Brasileiras. 2006. Tese (Doutorado) - Centro de Tecnologia Programa de Pós-Graduação em Engenharia Civil, Universidade Federal de Santa Catarina, Florianópolis, 2006.

WEN, T. J.; NOOR, H. C. S. Z. Z. Assessment of embodied energy and global warming potential of building construction using life cycle analysis approach: Case studies of residential buildings in Iskandar Malaysia. Energy and Buildings, v.93, p. 295 - 302. 2015. 